\title{
Water dissociation upon adsorption onto free iron clusters is size dependent
}

\author{
Denis M. Kiawi, ${ }^{1,2}$ Valeriy Chernyy, ${ }^{1}$ Jos Oomens, ${ }^{1,3}$ Wybren Jan Buma, ${ }^{3}$ \\ Zahra Jamshidi, ${ }^{4}$ Lucas Visscher, ${ }^{5}$ L.B.F.M. Waters,${ }^{6,2}$ and Joost M. Bakker ${ }^{1, *}$ \\ ${ }^{1}$ Radboud University, Institute for Molecules and Materials, FELIX \\ Laboratory, Toernooiveld 7, 6525 ED Nijmegen, The Netherlands \\ ${ }^{2}$ Anton Pannekoek Institute, University of Amsterdam, Science Park 904, 1098 XH Amsterdam, The Netherlands \\ ${ }^{3}$ Van 't Hoff Institute for Molecular Sciences, University of \\ Amsterdam, Science Park 904, 1098 XH Amsterdam, The Netherlands \\ ${ }^{4}$ Department of Physical Chemistry, Chemistry and Chemical Engineering \\ Research Center of Iran. P.O. Box 14335-186, Tehran, Iran \\ ${ }^{5}$ Amsterdam Center for Multiscale Modeling Section Theoretical Chemistry, VU \\ University Amsterdam, De Boelelaan 1083,1081 HV Amsterdam, The Netherlands \\ ${ }^{6}$ SRON Netherlands, Sorbonnelaan, Utrecht, The Netherlands
}

\begin{abstract}
Cationic iron clusters, produced through laser ablation and subsequently complexed with a water molecule $\mathrm{Fe}_{\mathrm{n}}{ }^{+}-\mathrm{H}_{2} \mathrm{O}(\mathrm{n}=6-15)$ are mass-selectively investigated via Infrared Multiple Photon Dissociation (IR-MPD) spectroscopy in the $300-1700 \mathrm{~cm}^{-1}$ spectral range. The experimental data are complemented by Density Functional Theory calculations at the OPBE/TZP level for the $\mathrm{Fe}_{13}{ }^{+}-\mathrm{H}_{2} \mathrm{O}$ system. For all cluster sizes, a large fraction of the studied clusters shows a resonance around the $1600 \mathrm{~cm}^{-1}$ frequency characteristic for the water bending vibration, indicating that water molecules remain intact upon binding to the cluster surface. However, in the far-IR, we find multiple absorption bands, which cannot be explained by molecularly adsorbed water only. DFT calculations for the $\mathrm{Fe}_{13}{ }^{+}-\mathrm{H}_{2} \mathrm{O}$ system predict molecular and dissociative adsorption to be exothermic; molecular adsorption is a barrierless process while the dissociative adsorption only requires $0.3 \mathrm{eV}$ activation energy, which can be overcome under the room-temperature conditions of our experiment. The observed spectrum can thus be explained by a mixture of clusters where for a majority water is adsorbed molecularly but for a small but significant fraction also dissociation of water molecules occurs. The bands observed at frequencies $300-700 \mathrm{~cm}^{-1}$ exhibit regular, sizedependent frequency shifts, showing that a) dissociation takes places on all cluster sizes and b) the interaction of water with the cluster surface is not influenced much by the particular cluster structure. The intensity evolution of the absorption bands suggests that dissociation is increasingly probable for larger cluster sizes
\end{abstract}

\section{INTRODUCTION}

The reactivity of metal nanoparticles can change dramatically from that of bulk metal surfaces, as exemplified by the enhanced catalytic activity of gold nanoparticles ${ }^{1}$. In the limit of clusters containing a few to a few tens of atoms, similar, a priori not expected, activities have been found for e.g. Fe. Bulk Fe has catalytic applications in many industrial processes including ammonia synthesis from nitrogen and hydrogen (the HaberBosch process $)^{2,3}$, paraffin synthesis from carbon monoxide and hydrogen (the Fischer-Tropsch process) ${ }^{4}$, and, more recently, in the production of single-wall carbon nanotubes ${ }^{5}$. In the cluster regime, $\mathrm{Fe}_{4}{ }^{+}$cations have been demonstrated to catalytically couple ethylenes to form benzene ${ }^{6}$. This observation and similar other studies have incited studies of the reactivity of iron clusters towards numerous simple molecules, see ref. ${ }^{7}$. and references therein for an extensive overview. Even before the demonstration of catalytic benzene formation, Riley and co-workers studied the adsorption of water on neutral Fe clusters, finding evidence for the temperature and size-dependent dissociation (lysis) of water followed by hydrogen evaporation ${ }^{8}$. Such results make Fe clusters a highly interesting subject to study, as they could offer novel routes towards (photo-)catalyzed water split- ting reactions to form molecular hydrogen.

The complicated electronic structure of Fe clusters, possessing a rich variety of possible spin states, has prevented a full theoretical evaluation of the reaction path and the relevant transition state barriers. Gutsev et al. ${ }^{9}$ theoretically studied the minimum energy structures for anionic, neutral and cationic Fe clusters with adsorbed water, explicitly considering the dissociation of water. They found in all cases that dissociation of water was energetically favorable by at least $0.5 \mathrm{eV}$, comparing favorably to the reported enthalpies of adsorption by Riley ${ }^{8}$. Moreover, the adsorption energies of water were all found to be higher than that of $\mathrm{H}_{2}$ (except for $\mathrm{Fe}_{4}{ }^{+}$), which is consistent with the observation of $\mathrm{Fe}_{n} \mathrm{O}$ products in the experiment. No reaction barrier energies were included in this work. These were calculated for the water lysis over dimers of a variety of transition metals, for which it was predicted that water lysis could take place under thermal conditions ${ }^{10}$. However, no barriers were calculated here for a subsequent $\mathrm{H}_{2}$ evolution.

Here, we present a combined experimental and theoretical study of the adsorption of water molecules onto cationic Fe clusters. Our motivation for this study is twofold. Firstly, we would like to find out the nature of the water adsorption product and to verify the predicted water lysis under thermal conditions. As the calculated 
$\mathrm{H}_{2}$ desorption energies for cationic $\mathrm{Fe}_{4}{ }^{+}$are higher than for water, the probability of finding a dissociated water molecule is deemed highest for the cations. Secondly, we recently investigated the structures of cationic cobalt clusters with an adsorbed water molecule through IR multiple-photon dissociation (IR-MPD) spectroscopy and found clear evidence for intact water by the presence of a band at frequencies characteristic for the water bending vibration ${ }^{11}$. The recorded IR spectra exhibited more bands at (lower) frequencies, where we expect vibrations involving motions between the cluster and the water molecule. The harmonic frequencies and intensities obtained from calculations provided a rather poor match, suggesting that a comparison with $0 \mathrm{~K}$ harmonic spectra is far from optimal for vibrations involving the rather flexible Co-water bond. It would therefore be highly interesting to see whether, in the case of an intact water adsorption product for $\mathrm{Fe}_{\mathrm{n}}{ }^{+}-\mathrm{H}_{2} \mathrm{O}$ clusters, spectral features similar to those in the chemically related $\mathrm{Co}_{\mathrm{n}}{ }^{+}-\mathrm{H}_{2} \mathrm{O}$ are observed.

To this purpose we present IR-MPD spectra of $\mathrm{Fe}_{\mathrm{n}}{ }^{+}-\mathrm{H}_{2} \mathrm{O}$ clusters for $\mathrm{n}=6-15$ and compare the spectra to DFT calculations. We limit the DFT calculations to $\mathrm{Fe}_{13}{ }^{+}-\mathrm{H}_{2} \mathrm{O}$ as it is not our purpose to perform an extensive search for structural isomers and because it is commonly assumed that $\mathrm{Fe}_{13}{ }^{+}$adopts an icosahedral geometry. Instead, our primary interest is to determine whether it is energetically plausible that water dissociates on cationic iron clusters at ambient temperatures, and to obtain spectral evidence for such a dissociation.

\section{METHODS}

\section{Experimental}

The experiments described in this paper are performed using a molecular beam instrument that is coupled to the Free Electron Laser for Infrared eXperiments (FELIX) ${ }^{12}$. Cationic iron clusters are created by pulsed laser ablation of an iron sample rod (Goodfellow, purity 99.99\%) using the second harmonic of a Nd:YAG laser. Ablation takes place in a $4 \mathrm{~mm}$ diameter flow tube type cluster growth channel in the presence of helium carrier gas that is introduced through a pulsed valve (General Valve, Series 9). The plasma generated and the carrier gas undergo multiple collisions leading to neutral, anionic and cationic clusters. In order to form cluster-water complexes, a mixture of $1 \%$ water vapor in helium is introduced 60 $\mathrm{mm}$ downstream into the flow tube channel before it is expanded into vacuum forming a molecular beam. The molecular beam passes through a $2 \mathrm{~mm}$ diameter skimmer (Beam Dynamics, Inc., model 2) to enter a differentially pumped vacuum chamber and is further shaped by a $1 \mathrm{~mm}$ aperture upon entering the extraction region of a reflectron time-of-flight mass spectrometer (R.M. Jordan TOF products, Inc.) in a third vacuum chamber. Here, the clusters interact with the IR laser beam that is aligned colinearly, but counter-propagating to the molecular beam. The focus of the IR beam lies about $25 \mathrm{~mm}$ before the extraction point and has a diameter of the same order as the molecular beam, thus ensuring that all extracted clusters have interacted with the IR laser beam.

IR light is produced by the Free-Electron Laser for Infrared eXperiments $\left(\right.$ FELIX) ${ }^{12}$. FELIX can produce IR radiation in the $66-3600 \mathrm{~cm}^{-1}$ spectral range but for the present experiments, only the $200-1700 \mathrm{~cm}^{-1}$ range is used. The FELIX temporal structure features 10 $\mu$ s duration macropulses at a repetition rate of 5 or $10 \mathrm{~Hz}$. The macropulse itself is a high-repetition $(1 \mathrm{GHz})$ series of picosecond duration laser pulses, so-called micropulses. The micropulses are transform-limited with a spectral bandwidth that can be adjusted to $0.2-1 \%$ RMS of the central frequency. In the current work the bandwidth was kept at $0.3 \%$. Typically employed macropulse energies range from 50-100 mJ.

A few $\mu$ s after interaction with FELIX, all cationic clusters are extracted by a set of pulsed high voltage plates into the mass spectrometer and detected with a microchannel plate detector. Whenever FELIX is in resonance with a vibrational mode of a given cluster, fragmentation is induced leading to the depletion of the number of detected $\mathrm{Fe}_{\mathrm{n}}{ }^{+}-\mathrm{H}_{2} \mathrm{O}$ cations. By varying the $\mathrm{FE}$ LIX frequency $\nu$ and recording the number of $\mathrm{Fe}_{\mathrm{n}}{ }^{+}-\mathrm{H}_{2} \mathrm{O}$ cations a depletion spectrum is obtained. To correct for long-term source fluctuations, the experiment is operated at twice the FELIX repetition rate, allowing for the recording of reference mass spectra in between successive FELIX pulses.

Experimental spectra for $\mathrm{Fe}_{\mathrm{n}}{ }^{+}-\mathrm{H}_{2} \mathrm{O}$ are obtained by carefully selecting a mass channel that has no contribution due to mono-oxide contaminations. As iron clusters are highly reactive to oxygen, any trace amount of oxygen will result in the formation of $\mathrm{Fe}_{\mathrm{n}} \mathrm{O}^{+}$clusters. The rich isotropic distribution of iron clusters will result in several overlapping peaks in the mass spectrum. However, each cluster size has mass peaks that are dominantly populated by $\mathrm{Fe}_{\mathrm{n}}{ }^{+}-\mathrm{H}_{2} \mathrm{O}$, e.g., $\mathrm{m} / \mathrm{z}=745$ and 746 for $\mathrm{Fe}_{13}{ }^{+}-\mathrm{H}_{2} \mathrm{O}$.

\section{Theoretical}

The Density Functional Theory (DFT) calculations reported here are performed using the Amsterdam Density Functional package (ADF2014) $)^{13-15}$. The calculations are carried out using the OPBE functional, which is a combination of Handy's OPTX modification of the Becke exchange functional with the Perdew-Burke-Ernzerhof (PBE) correlation functional ${ }^{16,17}$. The choice of functional is based on their excellent performance for the determination of the correct spin state for high/lowspin energy splitting in some previous studies of iron complexes $^{18-21}$. A triple $\zeta$ type (TZP) basis set from the ADF basis set library has been used in which the $1 \mathrm{~s}-2 \mathrm{p}$ 
core for $\mathrm{Fe}$ atoms and the 1 s for the $\mathrm{O}$ atoms have been kept frozen during the calculations. Geometry optimization has been performed without symmetry constraints. The treatment of transition metal clusters with DFT has proven especially challenging due to the large number of possible spin configurations. SCF convergence of high spin systems such as iron clusters is difficult and slightly relaxed SCF convergence criteria of $10^{-6}$ were tolerated.

The $\mathrm{Fe}_{13}{ }^{+}-\mathrm{H}_{2} \mathrm{O}$ cluster was chosen for DFT because a large body of previous theoretical work ${ }^{22-32}$ is available for iron clusters and indicates the high stability of near-icosahedral structures ${ }^{29,33}$. This exceptional stability is backed up by experimental data for this cluster size: collision induced dissociation experiments on $\mathrm{Fe}_{13}{ }^{+}$indicate a much higher dissociation energy for $\mathrm{Fe}_{13}{ }^{+}$than for other iron clusters ${ }^{34}$. Nevertheless, the right spin multiplicity of $\mathrm{Fe}_{13}{ }^{+}$still remains a topic of discussion. DFT studies on neutral $\mathrm{Fe}_{13}$ show the presence of two stable spin states $\left(M_{S}=34 / 2\right.$ and $\left.M_{S}=44 / 2\right)$ which depend on the orientation of the atoms in the cluster ${ }^{35}$. A review by Dunlap ${ }^{36}$ addresses the link between the spin multiplicity and the geometrical structure of a cluster. Several theoretical investigations on the cation yielded a minimum-energy spin state of $M_{S}=35 / 2 .{ }^{37-39}$ Structures with higher spin states have been found to be higher in energy. It is not our intention to enter into the debate on the magnetic structure of $\mathrm{Fe}_{13}{ }^{+}$; rather we will focus on the energetics and structure of its complex with water. As the experimental findings point at a spin moment of $M_{S}=35 / 2^{40-42}$, we employ this spin isomer.

The structure of bare $\mathrm{Fe}_{13}{ }^{+}$was calculated using the initial starting structures of the lowest energy conformers as proposed in previous studies ${ }^{29,33}$. Minima were verified by harmonic frequency calculations with analytical second derivatives. All energies reported here include zero-point energies. Once geometry and spin state of the bare cluster were established, a water molecule was added at several trial positions and the resulting structures reoptimized in a range of spin states around $M_{S}=35 / 2$. To establish whether the resulting structures are true minima, and, more importantly, to be able to analyze the experimental IR spectra, harmonic frequencies were calculated. The full reaction pathway for water adsorption and subsequent lysis over $\mathrm{Fe}_{13}{ }^{+}$was reconstructed, and transition states are verified by calculating harmonic frequencies. Coordinates and frequencies for all structures can be found in the SI; all frequencies reported in this paper refer to unscaled frequencies.

\section{RESULTS AND DISCUSSION}

Figure 1 displays mass spectra of $\mathrm{Fe}_{\mathrm{n}}{ }^{+}-\mathrm{H}_{2} \mathrm{O}$ clusters. Clusters containing up to $17 \mathrm{Fe}$ atoms are visible, along with up to two adsorbed water molecules. An enlarged fraction of the mass spectrum is shown in the lower panel. The black and red traces in the lower panel correspond to spectra as obtained in the presence (absence) of wa-

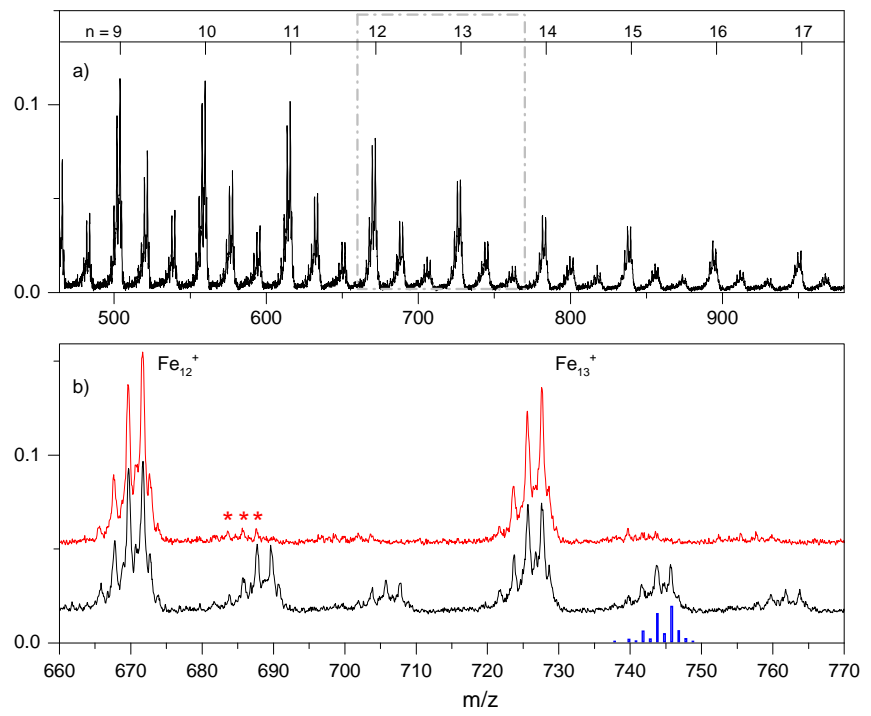

FIG. 1: Mass spectrum of $\mathrm{Fe}_{\mathrm{n}}-\mathrm{H}_{2} \mathrm{O}$ clusters ( $\left.\mathrm{n}=9-17\right)$ (top panel) and an enlarged portion of the mass spectrum (bottom panel) with (black) and without the presence of water in the reaction channel (red). The calculated isotopic distribution for $\mathrm{Fe}_{13}{ }^{+} \mathrm{H}_{2} \mathrm{O}$ is indicated by the blue stick spectrum.

ter in the reaction channel, respectively. In the absence of water, one can still observe minor contributions from $\mathrm{Fe}_{\mathrm{n}} \mathrm{O}^{+}$and $\mathrm{Fe}_{\mathrm{n}} \mathrm{O}_{2}{ }^{+}$clusters (indicated by asterisks) but these result from residual contaminant gases in the ablation source. In the presence of water, appreciable ion intensity from the bare iron clusters is transferred to the $\mathrm{Fe}_{\mathrm{n}}{ }^{+}-\mathrm{H}_{2} \mathrm{O}$ and $\mathrm{Fe}_{\mathrm{n}}{ }^{+}-\left(\mathrm{H}_{2} \mathrm{O}\right)_{2}$ mass channels. In the lower panel, the calculated isotopic distribution for $\mathrm{Fe}_{13}{ }^{+}-\mathrm{H}_{2} \mathrm{O}$ is shown. The excellent agreement between calculated and observed patterns indicate that the adsorption of water in our experiment does not lead to extra $\mathrm{Fe}_{\mathrm{n}} \mathrm{O}^{+}$formation. This could have been an indication for water lysis followed by the formation and desorption of $\mathrm{H}_{2}$, as suggested previously.

In Figure 2 the experimental IR-MPD spectra of $\mathrm{Fe}_{\mathrm{n}}{ }^{+}-\mathrm{H}_{2} \mathrm{O}$ clusters in the $200-1700 \mathrm{~cm}^{-1}$ spectral range are shown. The spectra are presented as the IRMPD intensity $I_{\text {IRMPD }}$ defined by

$$
I_{\mathrm{IRMPD}}=-\ln \left(\frac{I_{\mathrm{IR}}(\nu)}{I_{\mathrm{ref}}}\right)
$$

where $I_{\mathrm{IR}}(\nu)$ and $I_{\text {ref }}$ represent the ion intensities with and without irradiation by FELIX, respectively. $I_{\text {IRMPD }}(\nu)$ is further linearly corrected for the FELIX macropulse energy. Each spectrum shows several bands at the low frequencies $\left(300-700 \mathrm{~cm}^{-1}\right)$, and one band near $1600 \mathrm{~cm}^{-1}$. As the depletions in the lower-frequency part of the spectrum are substantially lower, their visibility has been enhanced by multiplying the IRMPD intensity by a factor four. The widths of the resonances are several tens of $\mathrm{cm}^{-1}$, which is considerably larger than the instrumental band width and is in fact indica- 


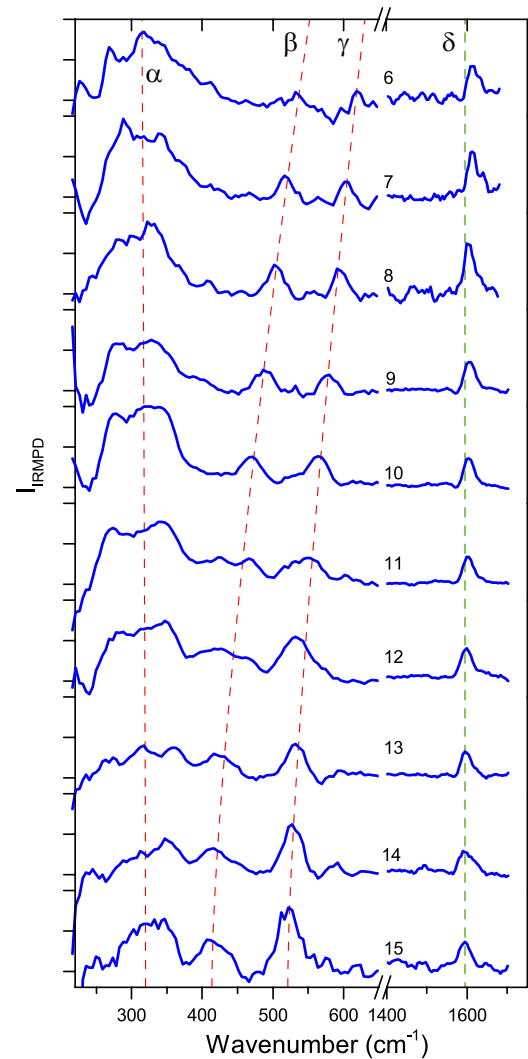

FIG. 2: IR multiple photon dissociation spectra of $\mathrm{Fe}_{n}{ }^{+}-\mathrm{H}_{2} \mathrm{O}$ $(\mathrm{n}=5-18)$ cluster complexes in the $200-1700 \mathrm{~cm}^{-1}$ spectral range. To clearly observe the vibrational structure in the 300$700 \mathrm{~cm}^{-1}$ range, intensities in this range have been multiplied by a factor of 4 . The size dependence of the bands is indicated with red dashed lines, labeled $\alpha, \beta, \gamma$ and $\delta$, that serve as guides to the eye.

tive of a multiple-photon excitation process ${ }^{43}$. In the spectral range between the two regions no resonances are observed.

The band close to $1600 \mathrm{~cm}^{-1}$, labeled $\delta$ in Figure 2, is directly diagnostic for molecular binding of water, as its frequency almost perfectly reproduces the frequency of the free water bending vibration at $1595 \mathrm{~cm}^{-144}$, indicated by the green dashed vertical line. At these frequencies, depletions of up to $80 \%$ of $\mathrm{I}_{\text {ref }}$ were observed, implying that the majority of species investigated contain an intact water molecule bound to the Fe cluster. It can be seen that the central frequency for this band $(\delta)$ is slightly blue-shifted with respect to the free water frequency, but that its frequency gradually decreases with cluster size $n$. This behaviour is consistent with what has been observed for water adsorption onto cationic vanadium ${ }^{45}$ and cobalt clusters $^{11}$

The deviations from the free water bending frequency are attributed to charge transfer interactions: once on the cluster surface, electron density on the oxygen atom is pulled towards the cluster thereby weakening the $\mathrm{O}-\mathrm{H}$ bond strength. For the larger clusters, such a red-shift is observed. The blue-shift for the smaller clusters might from this point of view appear counter intuitive. However, for the $\mathrm{Fe}^{+}$cation, it has been shown that charge transfer changes the $\mathrm{H}-\mathrm{O}-\mathrm{H}$ bond angle from its value in the free water molecule to 106.1 degree $^{46}$ and concurrently the bending frequency which by the same work was calculated to be at $1688 \mathrm{~cm}^{-1}$. The blue-shift observed for the smaller clusters is the result of these two effects.

In the lower-frequency range at least three distinct bands are observed for each cluster size. These bands show a rather regular size dependence, which is illustrated by the red dashed lines labeled $\alpha, \beta, \gamma$ in Figure 2 that serve as a guide to the eye. Such a size dependence is not unexpected as vibrations in this region involve the interactions between the cluster and the water molecule. However, the regularity of the size dependence strongly suggests that the frequencies of these bands are not very sensitive to the specific cluster structure.

DFT calculations have been used to elucidate the nature of the modes at lower frequencies and rationalize the observation of the intact water adsorption. The addition of water to either spin configuration of the optimized bare cluster appears to have no influence on their spin state. The $M_{S}=35 / 2$ species is still the minimum in the scan of possible spin states for $\mathrm{Fe}_{13}{ }^{+}-\mathrm{H}_{2} \mathrm{O}$. The preferred binding site for water on the clusters is a $\mu^{1}$ site, with the oxygen attached to the iron atom and the hydrogen atoms pointing away from the surface of the cluster (erect configuration). Trial structures where the $\mathrm{H}-\mathrm{O}-\mathrm{H}$ plane is oriented at smaller angles resulted in a reorientation of the water molecule to this erect configuration. This is in agreement with previous experimental studies where no evidence of a $\mu^{2}$ bridge binding site for $\mathrm{H}_{2} \mathrm{O}$ and $\mathrm{CO}$ on cobalt clusters was observed ${ }^{11,47}$. The calculations yield a binding energy of $0.44 \mathrm{eV}$ for the adsorbed water molecule, an energy that is readily available by the absorption of several resonant IR photons, and is thus fully in agreement with the loss of the water observed in our experiments. Also, it is satisfying to observe that this value is consistent with the reported experimental enthalpy of $0.5 \mathrm{eV}$ for binding of deuterated water to neutral $\mathrm{Fe}_{13}{ }^{8}$.

We further calculated the structures of different water binding motifs. Apart from the molecular binding, we found a partially and a fully dissociated water structure. The structure with a fully dissociated water molecule is found to be the lowest in energy, exothermic by 1.33 $\mathrm{eV}$ with respect to the reactants, while partially dissociated is exothermic by only $0.55 \mathrm{eV}$. These results are in good agreement with ab initio calculations performed by Bauschlicher and coworkers ${ }^{9}$, who also found a fully dissociated water complex to be the one of lowest energy. No structure with intact water was reported in that study.

In Figure 3 the experimentally obtained IRMPD spectrum is compared to calculated harmonic IR absorption spectra for $\mathrm{Fe}_{13}{ }^{+}$clusters with water bound in different motifs. The experiment shows at least six bands: the 


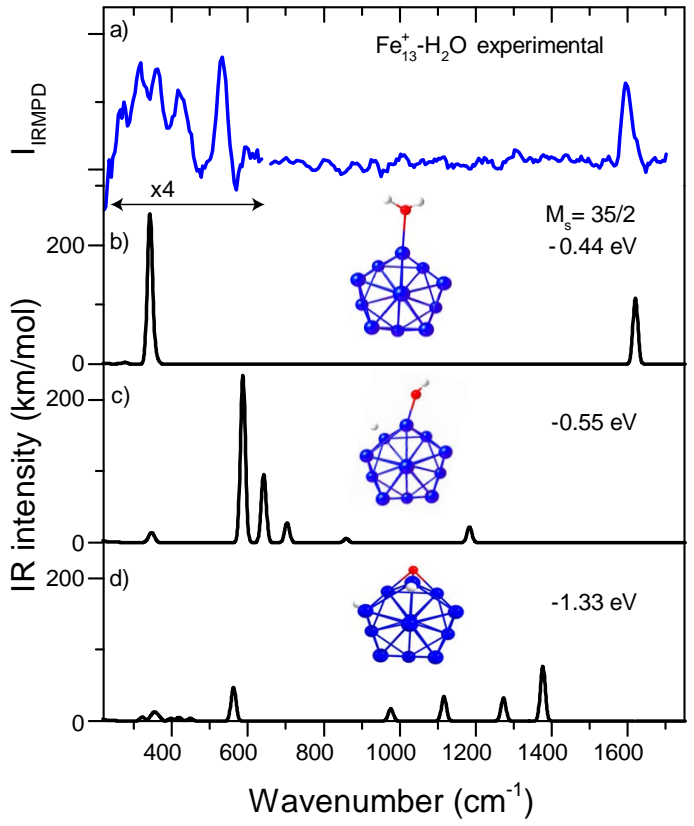

FIG. 3: Comparison of experimental IRMPD spectrum of $\mathrm{Fe}_{13}{ }^{+} \mathrm{H}_{2} \mathrm{O}$ with the harmonic frequencies for intact (molecular) binding (b), partially dissociated water (c) and fully dissociated water $(\mathrm{d})$.

water bending mode around $1600 \mathrm{~cm}^{-1}$, and five watercluster modes at $270,317,360,420$, and $530 \mathrm{~cm}^{-1}$, respectively. The bands at 360 and $420 \mathrm{~cm}^{-1}$ are part of a congested spectral structure comprising substructure with bands at 270 and $317 \mathrm{~cm}^{-1}$. In the spectral region below $700 \mathrm{~cm}^{-1}$, the most intense band predicted for the molecularly bound structure is the water libration mode, where the water molecule makes a wagging motion. We therefore assign this wagging mode as the main carrier of the congested structure. The width and shape of this structure strongly suggest that the underlying resonances are strongly saturated. This would also nicely explain the observation of more than a single band associated with the wagging mode: due to saturation on the fundamental transition combination bands will appear with enhanced relative intensities.

The pronounced band at $530 \mathrm{~cm}^{-1}$ is not predicted in the spectrum for molecularly adsorbed water. One could speculate that this is due to a combination mode. What argues against this, is that a combination band is expected to be considerably weaker than the fundamental. A more convincing assignment for the $530 \mathrm{~cm}^{-1}$ band is found in the spectrum for the hydroxylated cluster in panel c. Here, the low-frequency range is dominated by a band at $587 \mathrm{~cm}^{-1}$. This band is associated to the $\mathrm{Fe}-\mathrm{O}-\mathrm{H}$ bending vibration. The assignment of the $530 \mathrm{~cm}^{-1}$ experimental band to this fundamental vibration indicates that the water molecules on the clusters partly dissociate upon adsorption, which is consistent with the experimental observations for neutral iron clusters, where the loss of $\mathrm{H}_{2}$ is indicative for the dissociative

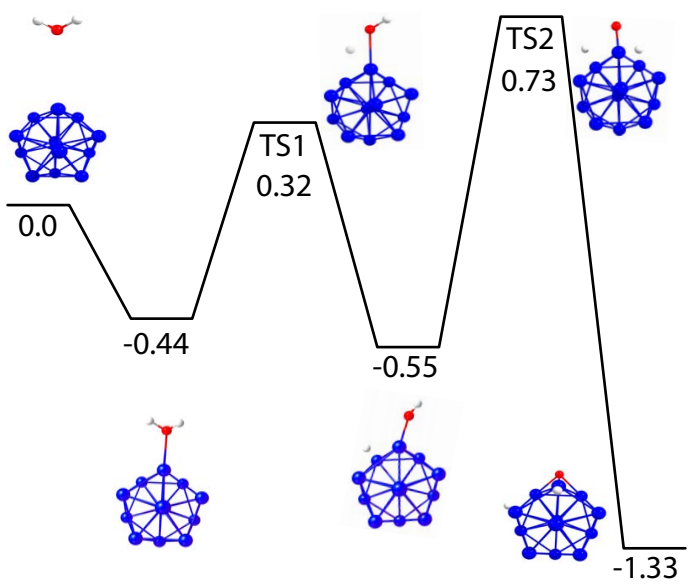

FIG. 4: Calculated reaction pathway for water adsorption and subsequent lysis over $\mathrm{Fe}_{13}{ }^{+}$. Energies are given in $\mathrm{eV}$, and include zero-point energy corrections.

adsorption ${ }^{8}$. We observe no other bands that could corroborate the finding of dissociated water; especially the predicted vibration at $1184 \mathrm{~cm}^{-1}$, the cluster-hydrogen stretch vibration is too weak to detect in the current experiment.

To reinforce our tentative assignment of the $530 \mathrm{~cm}^{-1}$ band to partly dissociated water, we constructed the reaction path identifying two transition states (TS), as shown in Figure 4. The first and second TS involve the transition from molecular water to a partially and to a fully dissociated water molecule on the surface with energy barriers of $0.32 \mathrm{eV}$ and $0.73 \mathrm{eV}$ with respect to the reactants, respectively. With such barriers it is indeed not unlikely that (partial) dissociation can take place under the employed near-ambient conditions: an estimate using a simple Arrhenius term approximation yields at room temperature a rate constant well within the experimental time window. Differential van der Waals corrections where assumed to be unimportant in this study as we consider the absorption and dissociation of a single water molecule. To verify this assumption we carried out calculations at the OPBE structures with the OPBD-D3 functional in which an explicit correction for van der Waals interaction is added ${ }^{48}$. The results are added to the SI and confirm this assumption: the water gets slightly stronger bound to the cluster by $0.04 \mathrm{eV}$ (an effect of $10 \%$ ) and the same slightly stronger bonding is preserved in the TS1 state, with the dispersion therefore having no effect on the relative energies of these states. To calibrate our results and estimate the influence of our choice of functional, we repeated the calculations ${ }^{10}$ on dissociation over $\mathrm{Fe}_{2}$. Using the current method, we find the adsorption product to be exothermic by $0.33 \mathrm{eV}$, and a barrier towards dissociation of $-0.09 \mathrm{eV}$ with respect to the reaction products i.e., $+0.24 \mathrm{eV}$ with respect to the adsorption product. This value is in excellent agreement with the reported free energy value of $0.27 \mathrm{eV}^{10}$ 
and provides additional support for the results for $\mathrm{Fe}_{13}{ }^{+}$.

Is water dissociation limited to $\mathrm{Fe}_{13}{ }^{+}$? As we have not calculated the structures of the other cluster sizes, we cannot make any firm conclusions. However, closer inspection of the spectra in Fig. 2 lead to two interesting observations. Firstly, we observe a highly regular pattern in the size dependence of the IR depletion spectra. As we have no more reason to expect other bands than the water wagging vibration for other cluster sizes than for $\mathrm{Fe}_{13}{ }^{+}$, it does not appear unreasonable to suspect that dissociation also takes place on other cluster sizes. Secondly, we note that the IR intensity of the water bending vibration and that of the band structure $\alpha$ close to 350 $\mathrm{cm}^{-1}$ are decreasing with cluster size, while the $\gamma$ band is exhibiting the opposite trend. Both observations are consistent with an increasing probability for water dissociation as a function of cluster size. These findings put a new light on our previous work on cobalt clusters, where a similar progression was observed. There, the dissociation mechanism was not investigated, but the present findings suggest that this route should be taken into account as well ${ }^{11}$.

In conclusion, we have recorded IR absorption spectra of cationic iron clusters complexed with water molecules in the $300-1700 \mathrm{~cm}^{-1}$ frequency range using IR-MPD spectroscopy. These data constitute the first experimental IR-MPD spectra of iron-water complexes where the iron-water intermolecular bonds are probed. Spectra for all iron clusters investigated show that water binds molecularly to iron clusters by the presence of a band at frequencies characteristic for the water bending vibration. At the same time, the far-IR part of the spectra gives evidence for the presence of partly dissociated water on all cluster sizes studied. DFT calculations for the $\mathrm{Fe}_{13}{ }^{+}$cluster suggest a dissociation pathway that is readily accessible at room temperature. Our findings are consistent with previous experiments, where dissociative adsorption was inferred from the loss of $\mathrm{D}_{2}$ upon the adsorption of two heavy water molecules ${ }^{8}$. That water binds dissociatively to iron is of course not altogether surprising. For bulk iron surfaces water is known to dissociate and to form hydroxyl, and only at elevated temperatures the water molecule is fully dissociated ${ }^{49-51}$. It is important to have established that water dissociates under these controlled conditions in a molecular beam. What is definitely very interesting is that the ratio of the IR absorption intensities indicate that there is a clear size dependence, and that dissociation is less likely to happen for smaller cluster sizes. For catalytic processes, where the difference between activation and poisoning can be important, such a size-tunable parameter may be of great importance.

\section{ACKNOWLEDGEMENTS}

We thank the entire FELIX staff, in particular Dr. A.F.G. van der Meer and Dr. B. Redlich for the skillful technical support. We gratefully acknowledge the Stichting voor Fundamenteel Onderzoek der Materie (FOM) for the support of the FELIX Laboratory. We thank Nederlandse Organisatie voor Wetenschappelijk Onderzoek Physical Sciences (NWO-EW) and the SurfSara Supercomputer Center for providing the computational resources. DMK acknowledges financial support from NWO Chemical Sciences as part of the Dutch Astrochemistry Network (grant 648.000.006). JO thanks the Stichting Physica for support.
* Electronic address: J.Bakker@science.ru.nl

1 M. Haruta, Catal. Today 36, 153 (1997).

2 J. E. Germain, Catalysis conversion of hydrocarbons, Academic press, New York (1969).

3 G. C. Bond, Catalysis by Metals, New York (1962).

${ }^{4}$ M. E. Dry, Catalysis Today 71, 227 (2002).

${ }^{5}$ S. Iijima and T. Ichihashi, Nature 363, 603 (1993).

${ }^{6}$ P. Schnabel, K. G. Weil, and M. P. Irion, Angew. Chem. Intl. Ed. 31, 636 (1992)

7 K. O. Fossan and E. Uggerud, Dalton Trans. pp. 892-897 (2004).

8 B. H. Weiller, P. S. Bechthold, E. K. Parks, L. G. Pobo, and S. J. Riley, J. Chem. Phys. 91, 4714 (1989).

9 G. Gutsev, M. Mochena, and C. Bauschlicher, Chem. Phys. Lett. 314, 291 (2005).

10 C. D. Zeinalipour-Yazdi and R. Van Santen, J. Phys. Chem. A 113, 6971 (2009).

11 D. M. Kiawi, J. M. Bakker, J. Oomens, W. J. Buma, Z. Jamshidi, L. Visscher, and L. B. F. M. Waters, J. Phys. Chem. A 119, 10828 (2015).

12 D. Oepts, A. van der Meer, and P. van Amersfoort, Infrared Phys. \& Techn. 36, 297 (1995).
${ }^{13}$ G. te Velde, F. M. Bickelhaupt, E. J. Baerends, C. Fonseca Guerra, S. J. A. van Gisbergen, J. G. Snijders, and T. Ziegler, J. Comp. Chem. 22, 931 (2001).

${ }^{14}$ C. Fonseca Guerra, J. G. Snijders, G. te Velde, and E. J. Baerends, Theor. Chim. Acta 99, 391 (1998).

15 ADF2013, Scm, theoretical chemistry, vrije universiteit, amsterdam, the netherlands, www.scm.com (2013).

16 N. C. Handy and A. J. Cohen, Mol. Phys. 99, 403 (2001).

17 J. P. Perdew, K. Burke, and M. Ernzerhof, Phys. Rev. Lett. 78, 1396 (1997).

18 M. Swart, A. R. Groenhof, A. W. Ehlers, and K. Lammertsma, J. Phys. Chem. A 108, 5479 (2004).

19 G. Ganzenmüller, N. Berkaïne, A. Fouqueau, M. E. Casida, and M. Reiher, J. Chem. Phys. 122, 234321 (2005).

20 J. Conradie and A. Ghosh, J. Chem. Theory Compu. 3, 689 (2007).

${ }^{21}$ K. Pierloot and S. Vancoillie, J. Chem. Phys. 128, 034104 (2008).

22 C. Yang, K. Johnson, D. Salahub, J. Kaspar, and R. Messmer, Phys. Rev. B 24, 5673 (1981).

23 M. Castro, Phys. Rev. B 47 (1993).

24 L. S. Wang, H. S. Cheng, and J. Fan, J. Chem. Phys. 24, 
9480 (1995).

25 L.-S. Wang, X. Li, and H.-F. Zhang, J. Chem. Phys. 262, $53(2000)$

26 A. Irigoras, M. D. C. Michelini, E. Sicilia, N. Russo, J. M. Mercero, and J. M. Ugalde, Chem. Phys. Lett. 376, 310 (2003).

27 G. L. Gutsev and C. W. Bauschlicher, J. Phys. Chem. A 107, 7013 (2003).

${ }^{28}$ G. L. Gutsev and C. W. Bauschlicher, J. Phys. Chem. A 107, 4755 (2003).

${ }^{29}$ G. Rollmann, P. Entel, and S. Sahoo, Comput. Mater. Sci. 35, 275 (2006).

30 Q.-M. Ma, Z. Xie, J. Wang, Y. Liu, and Y.-C. Li, Solid State Commun. 142, 114 (2007).

31 J. Hafner and D. Spišák, Phys. Rev. B 76, 094420 (2007).

${ }^{32}$ K. Cervantes-Salguero and J. M. Seminario, J. mol. model. 10, 1007 (2012)

33 C. Köhler, G. Seifert, and T. Frauenheim, Chem. Phys. 309, 23 (2005)

${ }^{34}$ L. Lian, C.-X. Su, and P. B. Armentrout, J. Chem. Phys. 97, 4072 (1992).

35 P. Bobadova-Parvanova, K. Jackson, S. Srinivas, and M. Horoi, Phys. Rev. B 66, 195402 (2002).

36 B. Dunlap, Phys. Rev. A 41, 5691 (1990).

37 G. L. Gutsev, C. A. Weatherford, P. Jena, E. Johnson, and B. R. Ramachandran, J. Phys. Chem. A 116, 10218 (2012).

38 M. Wu, A. K. Kandalam, G. L. Gutsev, and P. Jena, Phys. Rev. B 86, 174410 (2012).

39 H. K. Yuan, H. Chen, A. L. Kuang, C. L. Tian, and J. Z.
Wang, J. Chem. Phys. 139, 034314 (2013).

40 M. Niemeyer, K. Hirsch, V. Zamudio-Bayer, M. Langenberg, M. Kossick, C. Ebrecht, K. Egashira, A. Terasaki, T. Möller, B. von Issendorff, et al., Phys. Rev. Lett. 108, 1 (2012)

41 A. Langenberg, K. Hirsch, A. Lawicki, V. Zamudio-Bayer, M. Niemeyer, P. Chmiela, B. Langbehn, A. Terasaki, B. v. Issendorff, and J. T. Lau, Phys. Rev. B 90, 184420 (2014).

42 J. Meyer, M. Tombers, C. V. Wüllen, G. NiednerSchatteburg, S. Peredkov, W. Eberhardt, M. Neeb, S. Palutke, M. Martins, W. Wurth, et al., J. Chem. Phys. 104302 (2015).

43 J. Oomens, B. G. Sartakov, G. Meijer, and G. von Helden, Int. J. Mass Spectrom. 254, 1 (2006).

44 R. A. Toth, J. Mol. Spectr. 190, 379 (1998).

${ }^{45}$ T. Jaeger, A. Fielicke, G. von Helden, G. Meijer, and M. Duncan, Chem. Phys. Lett. 392, 409 (2004).

46 M. Trachtman, G. D. Markham, J. P. Glusker, P. George, and C. W. Bock, Inorg. Chem. 37, 4421 (1998).

47 A. Fielicke, G. von Helden, and G. Meijer, J. Chem. Phys. 128, 44308 (2006)

48 S. Grimme, J. Antony, S. Ehrlich, and H. Krieg, J. Chem. Phys. 132, 154104 (2010).

49 W. H. Hung, J. Schwartz, and S. L. Bernasek, Surf. Sci. 248, 332 (1991).

50 M. Henderson, Surf. Sci. Rep. 46, 1 (2002).

51 A. P. Grosvenor, B. A. Kobe, and N. S. McIntyre, Surf. Sci. 572, 217 (2004). 